\title{
Towards improving the healthcare services in least developed countries: a case of health needs assessment for telehealth in Yemen
}

\begin{abstract}
Motivated by the need to improve the healthcare system in least developed countries, this paper aims to investigate the need for utilizing aspects of Telehealth by rural health facilities in Yemen as a case that represents the least developed countries. A qualitative approach has been adopted in this paper. The review of documents taken from the Yemeni Ministry of Public Health, among others, revealed the lack of health facilities, professionals, equipment, and funds; inequity in the distribution of health facilities, professionals, and equipment between urban and rural; and lack of unified health information system and unified database. The observation revealed three categories of needs: needs in the provision of healthcare services, health professionalsôneeds; and needs in health management information. Analysis of the findings shows that Telehealth is appropriate solution and has the potential to improve the current health status by providing quality health services and exchange health information among rural areas in Yemen.
\end{abstract}

Keyword: Telehealth; Least developed countries; Health information system; Health information; Healthcare services; Health management information 